\title{
Spotted Fever Rickettsiosis in a Wildlife Researcher in Sabah, Malaysia: A Case Study
}

\author{
Milena Salgado Lynn ${ }^{1,2,3,4}$, Timothy William ${ }^{5}$ (i), Ampai Tanganuchitcharnchai ${ }^{6}$, \\ Suthatip Jintaworn ${ }^{7}$, Janjira Thaipadungpanit ${ }^{7}$, Mei Ho Lee ${ }^{8}$, Cyrlen Jalius ${ }^{1,3}$, \\ Peter Daszak ${ }^{8}$ (D), Benoît Goossens ${ }^{1,2,4,9}$, Tom Hughes ${ }^{8}$ and Stuart D. Blacksell ${ }^{6,7, *}$ (iD \\ 1 Danau Girang Field Centre, c/o Sabah Wildlife Department, Sandakan, Sabah 90009, Malaysia; \\ Salgado-LynnM@cardiff.ac.uk (M.S.L.); fielondra1988@gmail.com (C.J.); GoossensBR@cardiff.ac.uk (B.G.) \\ 2 School of Biosciences, Cardiff University, Cardiff CF10 3AX, UK \\ 3 Wildlife Health, Genetic and Forensic Laboratory, c/o Sabah Wildlife Department, \\ Kota Kinabalu, Sabah 88100, Malaysia \\ 4 Sustainable Places Research Institute, Cardiff University, Cardiff CF10 3BA, UK \\ 5 Jesselton Medical Centre, Kota Kinabalu, Sabah 88300, Malaysia; tim7008@gmail.com \\ 6 Centre for Tropical Medicine and Global Health, Nuffield Department of Clinical Medicine, \\ University of Oxford, Churchill Hospital, Oxford OX3 7FZ, UK; ampai@tropmedres.ac \\ 7 Mahidol-Oxford Tropical Medicine Research Unit, Faculty of Tropical Medicine, Mahidol University, \\ Bangkok 10400, Thailand; suthatip@tropmedres.ac (S.J.); Janjira@tropmedres.ac (J.T.) \\ 8 EcoHealth Alliance, New York, NY 10001-2320, USA; Lee@ecohealthalliance.org (M.H.L.); \\ daszak@ecohealthalliance.org (P.D.); tom.hughes@ecohealthalliance.org (T.H.) \\ 9 Sabah Wildlife Department, Kota Kinabalu, Sabah 88100, Malaysia \\ * Correspondence: stuart@tropmedres.ac; Tel.: +66-2-2036-3333
}

Received: 12 December 2017; Accepted: 28 February 2018; Published: 6 March 2018

\begin{abstract}
We present evidence for a case of spotted fever rickettsiosis with severe complications in a young adult male. Although spotted fever group rickettsiae (SFGR) have been reported as the most prevalent cause of rickettsiosis in rural areas of Sabah, Malaysia since the 1980s, this is the first detailed case report of suspected SFGR in the state. Current data on the prevalence, type, and thorough clinical reports on complications of SFGR and other rickettsioses in Sabah is lacking and required to raise the awareness of such diseases. There is a need to emphasize the screening of rickettsioses to medical personnel and to encourage the use of appropriate antibiotics as early treatment for nonspecific febrile illnesses in this region. Suspected rickettsioses need to be considered as one of the differential diagnoses for patients presenting with acute febrile illness for laboratory investigations, and early treatment instituted.
\end{abstract}

Keywords: rickettsiae; Malaysia; diagnosis

\section{Introduction}

Over the past 25 years, there has been an increase in the knowledge of rickettsial pathogens worldwide [1]. Nevertheless, there are still geographical areas where little information on rickettsioses is available regarding the clinical cases, the pathogens and the vectors [1-4]. Yet in Southeast Asia, and in travellers returning from this region, rickettsial diseases are among the leading causes of treatable acute febrile illnesses $[5,6]$. The clinical symptoms of rickettsioses may vary depending on the rickettsial species involved, and therefore diagnosis can be challenging even for experienced physicians [1]. Moreover, the specific laboratory methods for the diagnosis of infections are seldom available in remote areas, and even in large cities, in Southeast Asia [1,5,7]. In addition, the management of the disease could also be challenging given the (lack of) availability of the most commonly used antibiotics, the possible resistance to them, and the alternatives to such antibiotics [8,9]. 
Rickettsioses are considered endemic in Malaysia and mostly associated with rural/estate areas $[10,11]$ though current data on the prevalence and type of rickettsioses are scarce [12-16]. Reports of this disease in Sabah are limited to two retrospective serological studies in the early 2000s [13,14], one cross-sectional survey in 1986 [17], and one prospective study in 2017 [18]. In this report, we present evidence for a case of spotted fever rickettsioses in a wildlife researcher in Sabah, Malaysia, highlighting the need for increased awareness of rickettsioses as a cause of acute febrile illness in this region.

\section{Clinical History}

A previously-healthy 23-year-old British male presented to Jesselton Medical Centre (JMC), Kota Kinabalu, Sabah, Malaysia, with an acute febrile illness. The patient had been in Sabah for a period of three months prior to becoming ill. Earlier on the day of admittance at JMC the patient had attended a clinic from where he was discharged to wait two days for the results of diagnostic tests (dengue and malaria), evidently not recognising the severity of his symptoms. Upon admission to JMC (day 1, D1), the patient reported onset of symptoms four days prior, having high fever (over $39^{\circ} \mathrm{C}$ ), headache, dry cough, sweating, diarrhoea, joint and muscular pain, appetite loss, nausea, confusion and light-headedness. Physical examination indicated a mild rash of small, undefined, scattered red dots on the limbs and abdomen and mild haemoptysis, at which point he was admitted to the intensive care unit (ICU). Haematology and biochemistry results indicated severe thrombocytopenia, leukopenia, lymphopenia, and a mild cholestasis (Table 1). He was haemodynamically stable, his lungs were clear, and there was no evident hepatosplenomegaly. Following admission, the patient's condition rapidly deteriorated. The admission chest $\mathrm{X}$-ray was normal, but a repeat $\mathrm{X}$-ray the following day showed clear pleural effusion (Figure 1). On D3 post-admission, the patient went into hypotensive shock (BP 70-80/30-40). CT scans showed pleural effusion in the right lung as well as ground-glass opacities in the dependent portion of the lungs, bilaterally (Figure 1). Additionally, the liver and the spleen were enlarged at 21 and $15 \mathrm{~cm}$, respectively. Signs of abnormal liver function became evident on D5 post-admission and were persistent for more than two weeks (Table 1). Antibiotic therapy commenced with ceftriaxone $2 \mathrm{~g}$ o.d. and doxycycline $100 \mathrm{mg}$ b.d., with the former discontinued after the hypotensive shock on D3 post-admission, and the latter on D7 post-admission. Due to the patient's inability to take oral medication, the lack of diagnosis, and to ensure a broad coverage of illnesses, meropenem $1 \mathrm{~g}$ tds and azithromycin IV $500 \mathrm{mg}$ o.d. were started on D3 and D4 post-admission for four and seven days respectively; intravenous doxycycline, the preferred choice for this case, is not available in Malaysia. The patient became afebrile on D5 post-admission and subsequently improved over the following 2 weeks. He was discharged after 23 days hospitalization when he was fit to travel to his home country, where he made a full recovery.
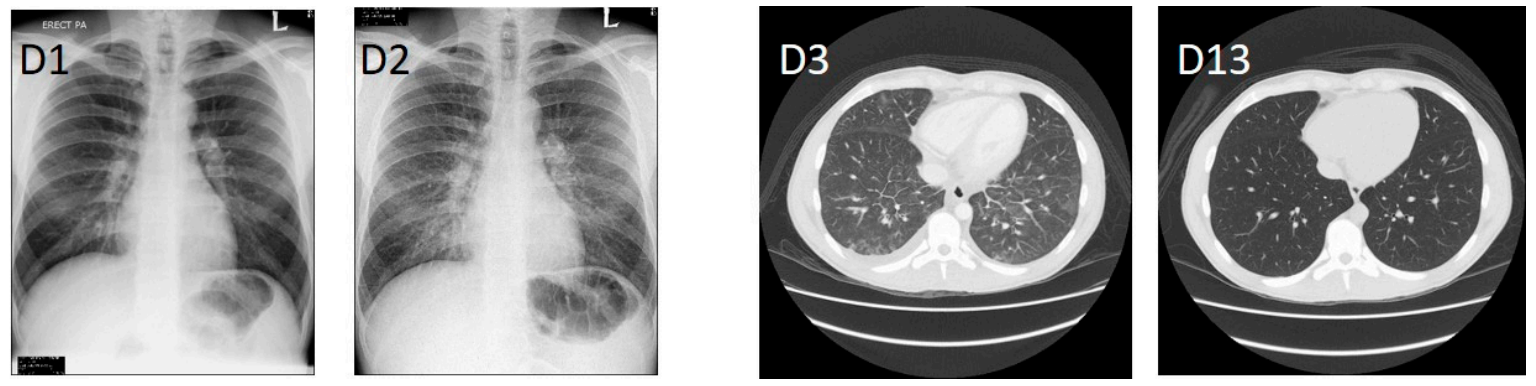

Figure 1. Chest X-rays upon admission (D1) and subsequent day (D2). Contrast-enhanced CT scan after oral and intravenous contrast administration at the day of hypotensive shock (D3), and post-treatment (D13). 
Table 1. Laboratory test results, showing thrombocytopenia and transaminitis that persisted for several days post-admission.

\begin{tabular}{cccccccc}
\hline Test & D1 * & D3 & D5 & D7 & D13 & D15 & Reference Range \\
\hline Haemoglobin, g/L & 156 & 132 & 132 & 125 & 140 & $\mathbf{1 2 9}$ & $130-180$ \\
White blood cells, cells/L & 4.6 & $\mathbf{3 . 8}$ & 6.6 & 10.1 & 7.6 & 5.8 & $4.0-11.0 \times 10^{9}$ \\
Lymphocytes, cells/L & $\mathbf{0 . 6}$ & $\mathbf{0 . 4}$ & 2.1 & $\mathbf{6 . 7}$ & $\mathbf{5 . 5}$ & $\mathbf{4 . 1}$ & $1.5-4.0 \times 10^{9}$ \\
Platelets, cells/L & $\mathbf{6 2}$ & $\mathbf{3 8}$ & $\mathbf{1 0 6}$ & 204 & $\mathbf{4 0 8}$ & 374 & $140-400 \times 10^{9}$ \\
Albumin, g/L & 39 & $\mathbf{3 0}$ & $\mathbf{2 4}$ & $\mathbf{2 6}$ & 38 & 38 & $35-52$ \\
Bilirubin, $\mu$ mol/L & $\mathbf{2 6}$ & 16 & 18 & 9 & 12 & 10 & $<21$ \\
Alkaline phosphatase, U/L & 84 & 74 & $\mathbf{1 2 5}$ & $\mathbf{1 3 6}$ & 114 & 100 & $30-120$ \\
Gamma-glutamyl transferase, U/L & $\mathbf{1 4 7}$ & $\mathbf{1 1 9}$ & $\mathbf{1 6 4}$ & $\mathbf{1 5 9}$ & $\mathbf{1 3 6}$ & $\mathbf{1 1 4}$ & $<50$ \\
Aspartate transferase, U/L & 37 & 43 & $\mathbf{2 0 6}$ & $\mathbf{8 8}$ & 38 & 33 & $<45$ \\
Alanine transaminase, U/L & 35 & 36 & $\mathbf{1 5 4}$ & $\mathbf{1 1 2}$ & $\mathbf{6 8}$ & 54 & $<55$ \\
C-reactive protein, mg/L & $\mathrm{N} / \mathrm{T}$ & $\mathrm{N} / \mathrm{T}$ & $\mathbf{9 3 . 9 9}$ & $\mathbf{3 5 . 3 4}$ & 2.55 & $\mathrm{~N} / \mathrm{T}$ & $>=5$ \\
\hline
\end{tabular}

${ }^{*}$ Admission day. N/T-not screened that day. Out-of-range values are highlighted in bold.

Prior to admission, the patient had been working in the forests of the Sandakan Division in Sabah for 20 days to perform ecological research in both urban and rural environments. At the field camps, he reported being in close proximity to cats and dogs, and occasionally with rats, and denied direct contact with wild vertebrates. The patient had multiple insect bites, including presumptive mosquito bites, but no tick bites, even though the patient and his team reported a large number of ticks in the last forest that was visited one week prior to the onset of the fever. Although attired in designated clothing for fieldwork, garments were not always immediately removed upon arrival at the base camps.

\section{Laboratory Diagnosis}

Initial laboratory investigations at JMC included dengue (IgM antibody and NS1 antigen), malaria (blood film), leptospirosis (IgM antibody and IgG antibody), and influenza A and B (virus A and virus $B$ antigens). All tests were negative. A multiplex PCR for 33 respiratory viruses and bacteria (FTD respiratory pathogens 33; Fast-Track Diagnostics) was positive for rhinovirus and also for influenza (A-F). An added tropical fever panel multiplex PCR (FTD Tropical Fever Core; Fast-Track Diagnostics) that included seven viral and bacterial families including Rickettsia spp. (targeting the citrate synthase gene, gltA) gave negative results for all pathogens. The multiplex PCRs were outsourced by JMC to a laboratory in West Malaysia. Additional throat, nasal, and rectal swabs, and urine were collected for PCRs, at the Sabah Wildlife Department's Wildlife Health, Genetic and Forensic Laboratory, on pooled cDNAs, screening for coronavirus [19,20], filovirus [21], influenza [22], paramyxovirus [23], enterovirus ([24], unpublished; VP4/2 and 5'UTR genes, Centre for Infection and Immunity, Columbia University, 2013), flavivirus [25], and hantavirus [26,27] using the PREDICT Universal Control. A PCR product was obtained from the enterovirus protocol [24] and confirmed by sequencing to be a $98.6 \%$ match to human rhinovirus A30 HRV30/DJ0692/11 (KC414928, 513 bp), which did not explain the patient's symptoms.

Rickettsiosis was suspected due to the patients' response to doxycycline and azithromycin therapy. Samples were tested retrospectively for genetic and serological evidence of rickettsial infection. Unfortunately, there was only a limited number of samples available for testing as the admission blood and serum samples were destroyed on D7. Additional samples were collected on D15 for PCR and serology testing. A real-time PCR for scrub typhus group (STG-Orientia tsutsugamushi), typhus group (TG—Rickettsia typhi and R. prowazekii) and SFGR (Rickettsia spp.) were all negative. An indirect micro-immunofluorescence assay (IFA) against STG (Orientia tsutsugamushi Karp, Kato and Gillam strains), TG (R. typhi Wilmington strain) and SFGR (R. honei and R. conorii) antigens was performed, with only SFGR positive (IgM 1:80, IgG 1:10). Serological examination of SFGR members $R$. honei (IgM 1:40, IgG 1:20), R. australis (IgM 1:40, IgG 1:40), R. conorii (IgM 1:40, IgG 1:20), R. felis (IgM 1:20, $\operatorname{IgG} 1: 10$ ) and $R$. massiliae (IgM 1:20, IgG 1:20), confirmed the result and indicated cross-reaction 
amongst the SFGR species. IFA results were confirmed by western blot (WB) [28] that demonstrated specific OmpA (130-160 kDa) and OmpB (115-150 kDa) bands for R. honei, R. australis and R. conorii, when reacted with the patient's serum using IgM and IgG conjugates (Figure 2).

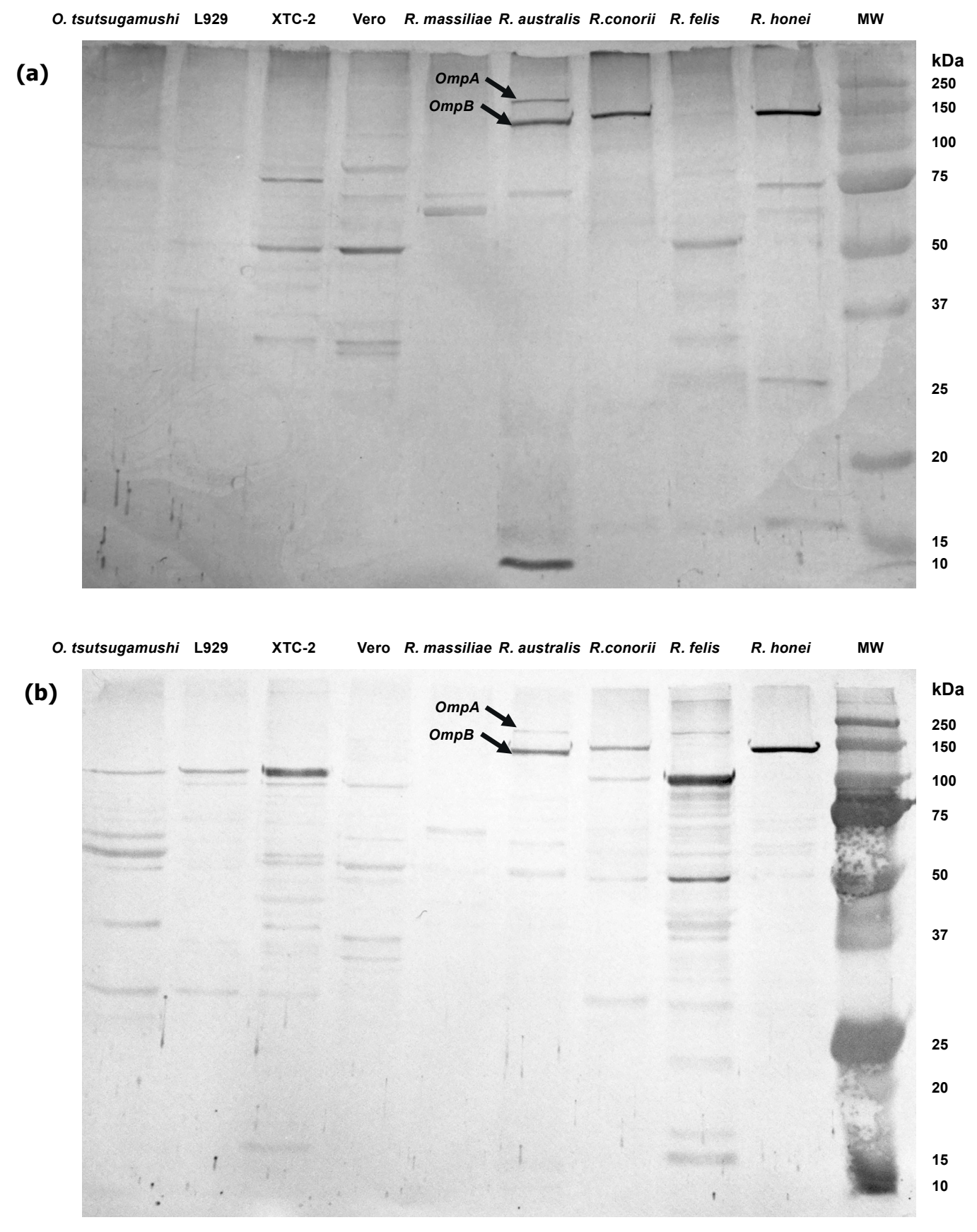

Figure 2. Western blot of the patient's serum reacted at a 1:80 dilution against host cell cultures and Orientia and Rickettsia spp. antigens using (a) IgM and (b) IgG conjugates. L929, Vero and XTC-2 are continuous cell lines used to propagate R. massiliae (Vero), R. conorii (L929), R. felis (XTC-2), R. honei (L929), R. australis (Vero) and Orientia tsutsugamushi (L929). MW = molecular weight marker in kiloDaltons (kDa).

\section{Discussion}

Based on the available evidence of response to doxycycline and azithromycin therapy and on the seropositivity demonstrated via IFA and WB tests against SFGR, there is evidence suggestive of 
SFG rickettsiosis in this patient. Since the patient was from a non-SFGR endemic region the relatively modest IFA titers may be consistent with a primary infection. Given that the sample was collected 15 days post-admission to hospital and 20 days post-onset of fever it was unlikely that the sample would be PCR positive. However, the PCR performed on the admission sample was negative, probably due to the lack of a whole blood pre-culturing step recommended by the manufacturer when typhoid is suspected. Therefore, the multiplex format performed might have lacked the sensitivity this case required. Also, the large variability of species belonging to the genus Rickettsia, and those yet to be identified in this region, might be another possibility [1,5]. The lateness of the sample collection and lack of early and follow-up samples is a weakness in this case study as there was no opportunity to demonstrate quantitative rise in antibody titer or to demonstrate the infectious agent via PCR.

Sabah and other similar areas of rural Malaysia are considered biodiversity hotspots and growing ecotourism destinations. There is greater involvement in outdoor activities in rural and/or remote areas, which may increase the contact with vector-borne pathogens. Additionally, shifts in land-use and deforestation have increased dramatically in Sabah during the last decades [29]. Combined, all these characteristics can be relevant to disease (re-)emergence, especially Rickettsia spp. For Sabah, the retrospective studies focused only on the health centre of Nabawan province of the Interior Division (rural), and on patients attending Queen Elizabeth Hospital (QEH) in the state capital (urban). The study of Tay et al. (2000), reported highest rickettsial seropositivity in Nabawan $(91.7 \%$; $n=145$ ) where $84.8 \%$ were SFGR ( $R$. honei, then TT118 strain) and $40 \%$ of the seropositive patients were agricultural workers. In another Sabah study, seven rural villages $(n=1220)$ in the Tawau and Kudat Divisions [17] demonstrated SFGR seropositivity four times higher in the two forest-dwelling populations of the Tawau Division (16.5\% each). Knowledge on the vectors and non-human hosts of rickettsial illnesses in Sabah is also limited. Ticks from rats collected in an island of the north of Sabah were all negative to SFGR by PCR [30]. However, $74 \%$ of fleas collected from domestic dogs in Sabah's West Coast Division were all positive to $R$. felis qPCR [31].

The diagnosis and treatment of rickettsioses in Sabah are highly challenging. First, rickettsial infections are probably more common than currently accepted. In 2015, the Malaysian Ministry of Health $(\mathrm{MMoH})$ reported only one case of typhoid fever nationwide [32]. However, the lack of routine testing capacity means formal $\mathrm{MMoH}$ notifications likely underestimate the true case incidence of rickettsial illnesses. For instance, a recently completed prospective study in northern Sabah demonstrates the common occurrence of rickettsioses among those with non-malarial acute febrile illness with an adjusted incidence of 6.5 cases/million for this state (a 23-fold increase from $\mathrm{MMoH}$ estimates) [18]. Second, in a region where dengue, malaria and leptospirosis (to name a few) are present, the lack of presence of a rash and/or an eschar can easily confound a physician who is not aware of rickettsiosis present in the area. Serology, in particular (micro)immunofluorescence assay, remains a gold standard for diagnosis, even when it has already been suggested that this technique should only be considered in areas with previously established seroprevalence [1,33], something that has not been done in Sabah. Moreover, the experienced manpower needed to perform the tests and to minimize the misinterpretation of results is, in most cases, lacking. In addition, specific equipment is also needed and it is often unavailable in the rural/estate areas, where these diseases are more likely to be present in Sabah. Nucleic acid methods present the same limitations as serology in such regions. Even in urban areas, serology may be adequate for diagnosis of spotted fever rickettsiosis, but the etiologic agent is not likely to be identified [1]. To reduce the delay in diagnosis, a rapid antigen-based diagnostic test produced locally would be desirable [7]. Third, doxycycline remains the standard treatment for SFG and TG rickettsioses [9]. Given that rickettsioses are endemic in Malaysia, it would be more than appropriate to have intravenous doxycycline available for treatments where patients are acutely ill. Although meropenem has not been commonly used to treat rickettsioses [9], it proved useful in this case and should be considered for this type of disease in Malaysia.

To conclude, there should be an emphasis on clinicians in Sabah to closely monitor patients with acute febrile illness given the historical and current prevalence of rickettsioses and the rapid and 
unpredictable fatal course of spotted typhus. Special attention must be given to those patients with glucose-6-phosphate dehydrogenase (G6PD) deficiency, a condition present in five to ten percent of all ethnic races in Sabah [34,35], since rapid fulminant courses of SFGR is known to occur in patients with this condition [36]. Therefore, typhus and typhus-like diseases must be included in the early differential diagnosis of nonspecific fevers in Sabah. In addition, a culture of preserving acute and convalescent samples for repetition of tests to confirm diagnoses must also be encouraged. Finally, without a rapid and reliable diagnostic test for confirming or excluding the disease at an early stage, the prompt administration of empirical antibiotic therapy to patients presenting symptoms related to rickettsioses is recommended in a closely-supervised manner to prevent antibiotic resistance, which will be detrimental at the individual and community level [8,37].

Acknowledgments: Stuart Blacksell is funded by the Wellcome Trust of Great Britain. The use of the PREDICT Universal Control was made possible by the generous support of the American people through the United States Agency for International Development (USAID) Emerging Pandemic Threats PREDICT project (cooperative agreement number GHN-A-OO-09-00010-00).5. We are grateful to Matthew Grigg for his comments on rickettsioses in Sabah during the early stages of the manuscript.

Author Contributions: M.S.L. and T.W. contributed to the manuscript conceptualization. M.S.L. contributed to the review and analysis of literature, and original draft preparation of the manuscript. T.W. treated the patient, facilitated the acquisition of additional non-acute samples, and shared the initial laboratory results from J.M.C. or those outsourced by J.M.C., C.J. liaised with J.M.C. for sample aliquoting, performed DNA extractions on the samples, and prepared the samples and DNA for transportation and export. A.T., S.J. and J.T. performed all serological tests under the supervision of S.D.B., M.H.L. tested the samples for virus using the PREDICT Universal Control. T.H., P.D., B.G. and S.D.B. provided funding and resources. S.D.B. scrutinized and edited the manuscript. M.S.L. and S.D.B. reviewed and corrected the paper. All authors reviewed and approved the manuscript.

Conflicts of Interest: The authors declare no conflict of interest.

\section{References}

1. Parola, P.; Paddock, C.D.; Socolovschi, C.; Labruna, M.B.; Mediannikov, O.; Kernif, T.; Abdad, M.Y.; Stenos, J.; Bitam, I.; Fournier, P.E.; et al. Update on tick-borne rickettsioses around the world: A geographic approach. Clin. Microbiol. Rev. 2013, 26, 657-702. [CrossRef] [PubMed]

2. Parola, P.; Paddock, C.D.; Raoult, D. Tick-borne rickettsioses around the world: Emerging diseases challenging old concepts. Clin. Microbiol. Rev. 2005, 18, 719-756. [CrossRef] [PubMed]

3. Parola, P.; Raoult, D. Ticks and tickborne bacterial diseases in humans: An emerging infectious threat. Clin. Infect. Dis. 2001, 32, 897-928. [CrossRef] [PubMed]

4. Walker, D.H. Rickettsiae and rickettsial infections: The current state of knowledge. Clin. Infect. Dis. 2007, 45, S39-S44. [CrossRef] [PubMed]

5. Aung, A.K.; Spelman, D.W.; Murray, R.J.; Graves, S. Review article. Rickettsial infections in Southeast Asia: Implications for local populace and febrile returned travelers. Am. J. Trop. Med. Hyg. 2014, 91, 451-460. [CrossRef] [PubMed]

6. Jensenius, M.; Han, P.V.; Schlagenhauf, P.; Schwartz, E.; Parola, P.; Castelli, F.; Von Sonnenburg, F.; Loutan, L.; Leder, K.; Freedman, D.O. Acute and potentially life-threatening tropical diseases in western travelers-A GeoSentinel multicenter study, 1996-2011. Am. J. Trop. Med. Hyg. 2013, 88, 397-404. [CrossRef] [PubMed]

7. Wilde, H.; Suankratay, C. There is need for antigen-based rapid diagnostic tests to identify common acute tropical illnesses. J. Travel Med. 2007, 14, 254-258. [CrossRef] [PubMed]

8. Kelly, D.; Fuerst, P.; Richards, A. The historical case for and the future study of antibiotic-resistant scrub typhus. Trop. Med. Infect. Dis. 2017, 2, 63. [CrossRef]

9. Botelho-Nevers, E.; Socolovschi, C.; Raoult, D.; Parola, P. Treatment of Rickettsia spp. infections: A review. Expert Rev. Anti-Infect. Ther. 2012, 10, 1425-1437. [CrossRef] [PubMed]

10. Marchette, N.J. Rickettsioses (tick typhus, Q-fever, urban typhus) in Malaya. J. Med. Entomol. 1966, 2, 339-371. [CrossRef] [PubMed]

11. Tee, T.S.; Kamalanathan, M.; Suan, K.A.; Chun, S.S.; Ming, H.T.; Yasin, R.M.; Devi, S. Seroepidemiologic survey of Orientia tsutsugamushi, Rickettsia typhi, and TT118 spotted fever group rickettsiae in rubber estate workers in Malaysia. Am. J. Trop. Med. Hyg. 1999, 61, 73-77. [CrossRef] [PubMed] 
12. Tay, S.T.; Kamalanathan, M.; Rohani, M.Y. Antibody prevalence of Orientia tsutsugamushi, Rickettsia typhi and TT118 spotted fever group rickettsiae among Malaysian blood donors and febrile patients in the urban areas. Southeast Asian J. Trop. Med. Public Health 2003, 34, 165-170. [PubMed]

13. Tay, S.T.; Rohani, M.Y. The use of the indirect immunoperoxidase test for the serodiagnosis of rickettsial diseases in Malaysia. Southeast Asian J. Trop. Med. Public Health 2002, 33, 314-320. [PubMed]

14. Tay, S.T.; Ho, T.M.; Rohani, M.Y.; Devi, S. Antibodies to Orientia tsutsugamushi, Rickettsia typhi and spotted fever group rickettsiae among febrile patients in rural areas of Malaysia. Trans. R. Soc. Trop. Med. Hyg. 2000, 94, 280-284. [CrossRef]

15. Kho, K.L.; Koh, F.X.; Singh, H.K.L.; Zan, H.A.M.; Ponnampalavanar, S.; Kukreja, A.; Tay, S.T. spotted fever group rickettsioses and murine typhus in a Malaysian teaching hospital. Am. J. Trop. Med. Hyg. 2016, 95, 765-768. [CrossRef] [PubMed]

16. Sagin, D.D.; Ismail, G.; Nasian, L.M.; Jok, J.J.; Pang, E.K.H. Rickettsial infection in five remote Orang Ulu villages in upper Rejang River, Sarawak, Malaysia. Southeast Asian J. Trop. Med. Public Health 2000, 31, 733-735. [PubMed]

17. Taylor, A.C.; Hii, J.; Kelly, D.J.; Davis, D.R.; Lewis, G.E. A serological survey of scrub, tick, and endemic typhus in Sabah, East Malaysia. Southeast Asian J. Trop. Med. Public Health 1986, 17, 613-619. [PubMed]

18. Reller, M.E.; Grigg, M.; William, T.; Yeo, T.; Clemens, E.G.; Dumler, J.S. Rickettsial infections as a major etiology of acute febrile illness: A prospective study in northern Sabah, Borneo, East Malaysia [abstract]. In: American Society of Tropical Medicine and Hygiene Sixty-Sixth Annual Meeting, 2017 November 5-9; Baltimore, Maryland, USA; abstract number 1982. Am. J. Trop. Med. Hyg. 2017, 97, 1-674. [CrossRef]

19. Watanabe, S.; Masangkay, J.S.; Nagata, N.; Morikawa, S.; Mizutani, T.; Fukushi, S.; Alviola, P.; Omatsu, T.; Ueda, N.; Iha, K.; et al. Bat coronaviruses and experimental infection of bats, the Philippines. Emerg. Infect. Dis. 2010, 16, 1217-1223. [CrossRef] [PubMed]

20. Quan, P.L.; Firth, C.; Street, C. Identification of a severe acute respiratory syndrome coronavirus-like virus in a leaf-nosed bat in Nigeria. mBio 2010, 1, e00208-10. [CrossRef] [PubMed]

21. Zhai, J.; Palacios, G.; Towner, J.S.; Jabado, O.; Kapoor, V.; Venter, M.; Grolla, A.; Briese, T.; Paweska, J.; Swanepoel, R.; et al. Rapid molecular strategy for filovirus detection and characterization. J. Clin. Microbiol. 2007, 45, 224-226. [CrossRef] [PubMed]

22. Anthony, S.J.; Leger, J.A.S.; Pugliares, K.; Ip, H.S.; Chan, J.M.; Carpenter, Z.W.; Navarrete-Macias, I.; Sanchez-Leon, M.; Saliki, J.T.; Pedersen, J.; et al. Emergaence of fatal avian influenza in New England harbour seals. mBio 2012, 3, e00166-12. [CrossRef] [PubMed]

23. Tong, S.; Chern, S.W.W.; Li, Y.; Pallansch, M.A.; Anderson, L.J. Sensitive and broadly reactive reverse transcription-PCR assays to detect novel paramyxoviruses. J. Clin. Microbiol. 2008, 46, 2652-2658. [CrossRef] [PubMed]

24. Allan Nix, W.; Oberste, M.S.; Pallansch, M.A. Sensitive, seminested PCR amplification of VP1 sequences for direct identification of all enterovirus serotypes from original clinical specimens. J. Clin. Microbiol. 2006, 44, 2698-2704. [CrossRef]

25. Moureau, G.; Temmam, S.; Gonzalez, J.P.; Charrel, R.N.; Grard, G.; de Lamballerie, X. A real-time RT-PCR method for the universal detection and identification of flaviviruses. Vector-Borne Zoonotic Dis. 2007, 7, 467-478. [CrossRef] [PubMed]

26. Aitichou, M.; Saleh, S.S.; McElroy, A.K.; Schmaljohn, C.; Ibrahim, M.S. Identification of Dobrava, Hantaan, Seoul, and Puumala viruses by one-step real-time RT-PCR. J. Virol. Methods 2005, 124, 21-26. [CrossRef] [PubMed]

27. Raboni, S.M.; Rubio, G.; De Borba, L.; Zeferino, A.; Skraba, I.; Goldenberg, S.; Dos Santos, C.N. Clinical survey of hantavirus in southern Brazil and the development of specific molecular diagnosis tools. Am. J. Trop. Med. Hyg. 2005, 72, 800-804. [PubMed]

28. Stenos, J.; Ross, B.; Feng, H.M.; Crocquet-Valdes, P.; Walker, D. Protein characterization of Australian spotted fever group rickettsiae and monoclonal antibody typing of Rickettsia honei. J. Clin. Microbiol. 1997, 35, 261-263. [PubMed]

29. Reynolds, G.; Payne, J.; Sinun, W.; Mosigil, G.; Walsh, R.P.D. Changes in forest land use and management in Sabah, Malaysian Borneo, 1990-2010, with a focus on the Danum Valley region. Philos. Trans. R. Soc. B Biol. Sci. 2011, 366, 3168-3176. [CrossRef] [PubMed] 
30. Adrus, M.; Ahamad, M.; Abdullah, M.T. Detection of rickettsiae in engorged ticks from small mammals in Malaysia. Borneo J. Resour. Sci. Technol. 2014, 4, 34-41.

31. Kernif, T.; Socolovschi, C.; Wells, K.; Lakim, M.B.; Inthalad, S.; Slesak, G.; Boudebouch, N.; Beaucournu, J.C.; Newton, P.N.; Raoult, D.; et al. Bartonella and Rickettsia in arthropods from the Lao PDR and from Borneo, Malaysia. Comp. Immunol. Microbiol. Infect. Dis. 2012, 35, 51-57. [CrossRef] [PubMed]

32. Health Informatics Centre. Health Indicators 2016: Indicators for Monitoring and Evaluation of Strategy Health for All; MOH/S/RAN/18.16(AR); Health Informatics Centre, Planning Division, Ministry of Health Malaysia: Putrajaya, Malaysia, 2016.

33. Blacksell, S.D.; Bryant, N.J.; Paris, D.H.; Doust, J.A.; Sakoda, Y.; Day, N.P.J. Scrub typhus serologic testing with the indirect immunofluorescence method as a diagnostic gold standard: A lack of consensus leads to a lot of confusion. Clin. Infect. Dis. 2007, 44, 391-401. [CrossRef] [PubMed]

34. Fong, T. Prevalence of erythrocyte G6PD deficiency in Sabah. Mod. Med. Asia 1977, 13, 14-16. [PubMed]

35. Khoo, K.K. The treatment of malaria in glucose-6-phosphate dehydrogenase deficient patients in Sabah. Ann. Trop. Med. Parasitol. 1981, 75, 591-595. [CrossRef] [PubMed]

36. Rathi, N.; Rathi, A. Rickettsial infections: Indian perspective. Indian Pediatr. 2010, 47, 157-164. [CrossRef] [PubMed]

37. Bell, B.G.; Schellevis, F.; Stobberingh, E.; Goossens, H.; Pringle, M. A systematic review and meta-analysis of the effects of antibiotic consumption on antibiotic resistance. BMC Infect. Dis. 2014, 14, 1-25. [CrossRef] [PubMed]

(C) 2018 by the authors. Licensee MDPI, Basel, Switzerland. This article is an open access article distributed under the terms and conditions of the Creative Commons Attribution (CC BY) license (http:/ / creativecommons.org/licenses/by/4.0/). 